\title{
Genus Pullimosina (Diptera: Sphaeroceridae) in China with description of a new species
}

\author{
Li-Xin Su, Guang-Chun Liu* \& Jie Xu
}
Su, L.-X., Liu, G.-C. \& Xu, J. 2013: Genus Pullimosina (Diptera: Sphaero- ceridae) in China with description of a new species. - Entomol. Fennica 24: $1-8$.

The genus Pullimosina Roháček is revised to have four species in China, including a new species, Pullimosina (Dahlimosina) quadripulata sp. n. For Pullimosina (Pullimosina) heteroneura (Haliday) a new record for Continental China is reported. All males of the four species and the female of the new species are described and illustrated. A key to these species is presented.

L.-X. Su \& G.-C. Liu, Liaoning Key Laboratory of Urban Integrated Pest Management and Ecological Security, Shenyang University, Shenyang, Liaoning 110044, China; *corresponding author'se-mail:liugc@yahoo.cn J. Xu, Shenyang Normal University, Shenyang, Liaoning 110034, China

Received 5 June 2012, accepted 10 August 2012

\section{Material and methods}

\section{Introduction}

Pullimosina Roháček, 1983 occurs in all zoogeographic regions of the world and comprises 28 species, the majority of which are from the Holarctic Region (Roháček et al. 2001, Hayashi 2006, Marshall et al. 2011). Only two species of Pullimosina are known from China (Su 2011).

While checking a series of Pullimosina specimens in the collections of Liaoning Key Laboratory of Urban Integrated Pest Management and Ecological Security, Shenyang University, Shenyang, Liaoning, China, we found one further undescribed species, which is superficially similar to Pullimosina (Dahlimosina) yukonensis Marshall, 1986. The genus Pullimosina is here expanded to include two additional species in China: Pullimosina (Dahlimosina) quadripulata sp. n. and Pullimosina (Dahlimosina) heteroneura.
The examined specimens were collected from mixed forests with sweep net and Malaise trap and preserved in alcohol. All studied specimens were preserved in Liaoning Key Laboratory of Urban Integrated Pest Management and Ecological Security, Shenyang University, China. The descriptions and illustrations were prepared from specimens or their dissected parts in ethanol or glycerine.

Morphological terminology follows Smith and Marshall (2004).

\section{Taxonomy}

\subsection{Pullimosina (Dahlimosina) quadripulata Su, sp. n. (Figs. 1, 2)}

Type material. Holotype $\delta^{\Uparrow}$ : China, Liaoning Province, Benxi City, Yanghugou, 12439' 48.4'E, $41^{\circ} 13$ '43.2'N, 13.VII.2008 Jianfeng Wang leg. 

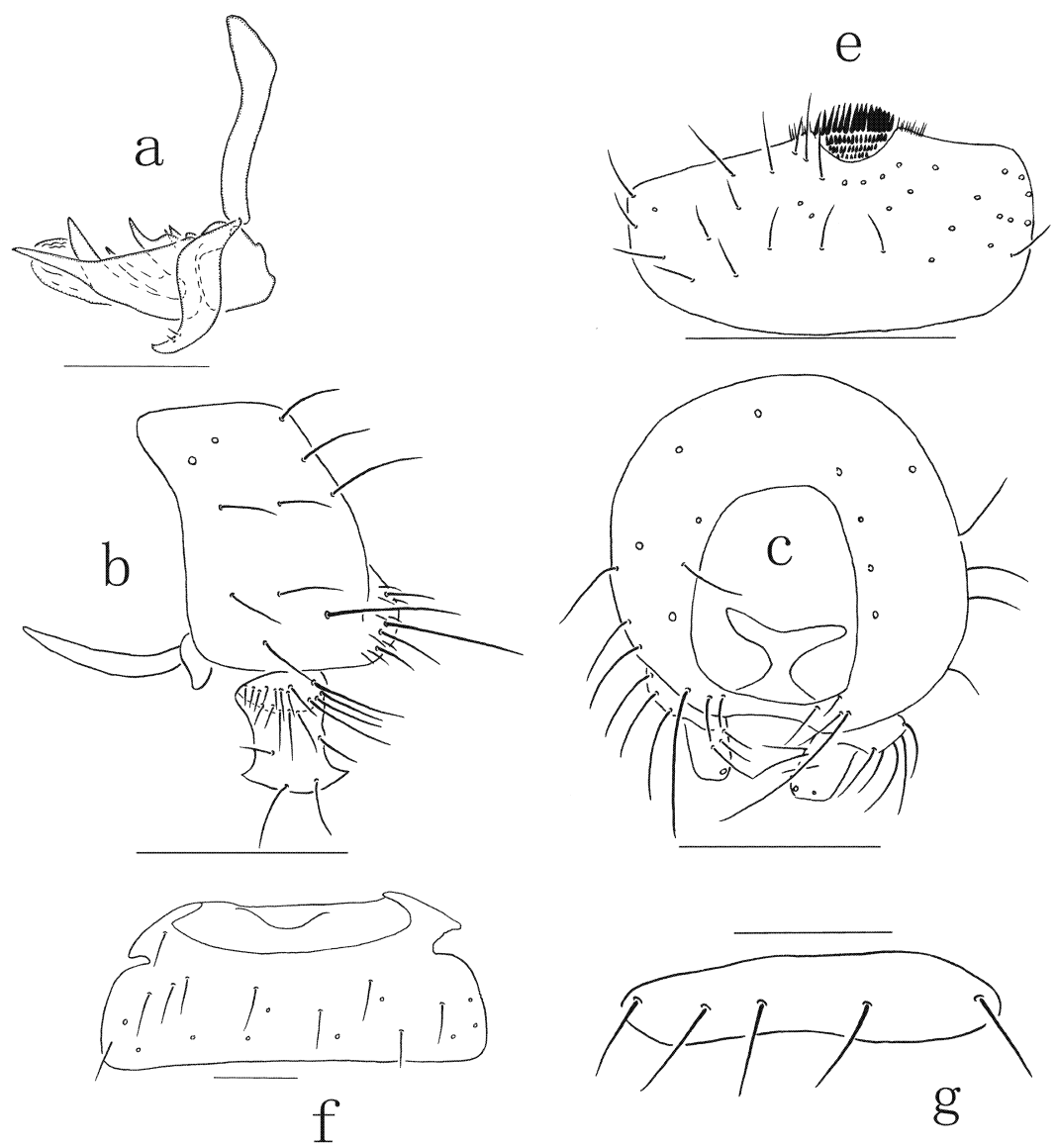

Fig. 1. Pullimosina (Dahlimosina) quadripulata Su, sp. n., holotype male, from Liaoning, China. - a. Aedeagus and associated parts laterally. - b. Genitalia laterally. - c. Genitalia caudally. -d. Surstylus laterally.
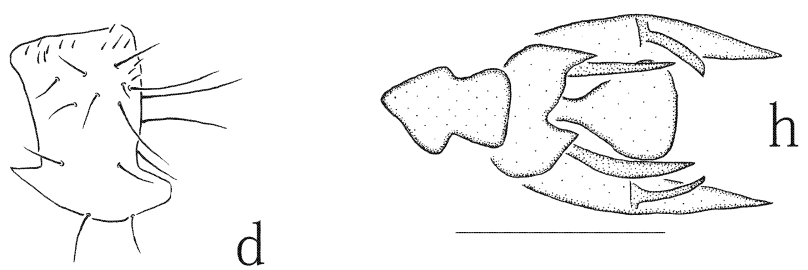

- e. Sternite 5 ventrally. - f. Syntergite 1+2 ventrally. - g. Synsternite $1+2$ ventrally. $-h$. Aedeagus and associated parts, dorsally. Scale bars $0.1 \mathrm{~mm}$.

Paratypes $1 \delta^{\lambda}$ : China, Liaoning Province, Benxi City, Yanghugou, 124³9'46.5' $\mathrm{E}, \quad 41^{\circ} 15^{\prime}$ ' 41.2”N, 14.VII.2010 Jianfeng Wang leg.; 2 ઠึે, 1 q: China, Liaoning Province, Benxi City, Yanghugou, $124^{\circ} 39^{\prime} 48.4^{\prime \prime} \mathrm{E}, \quad 41^{\circ} 13^{\prime} 43.2^{\prime \prime} \mathrm{N}$, 13.VII.2008 Jianfeng Wang leg.

Description. Body length $1.4-1.8 \mathrm{~mm}$, wing length $1.3-1.6 \mathrm{~mm}$. General colour shining black, including head; halter with stem pale brown, knob black; legs almost black except for trochanters, apices of femora, basal parts of tibiae and tarsi, darker brown. Head: Only with 1 pair of upcurved setulae behind ocellar triangle. Interfrontal setae in 3 pairs, sbuequal in length. A min- ute pair of short setulae below and outside interfrontals also present. Eye height about 2.4 times as long as genal height. Aristal hairs twice as long as basal width of arista. Genal seta small, but indistinct, whole gena almost completely covered by small setulae.

Thorax: Thoracic chaetotaxy: 1 long postpronotal seta; 2 dorsocentral setae, anterior very short, almost on the suture, prescutellar dorsocentral seta slightly shorter than scutellar length; acrostichal setulae in 8 rows in front of suture. Katepisternum with 1-2 anterior small setulae to posterior seta. Scutellum with 4 marginal setae, 2-3 small setulae in front of laterobasal scutellar 
Fig. 2. Pullimosina (Dahlimosina) quadripulata Su, sp. n., paratype female, from Liaoning, China. - a. Synsternite $1+2$ ventrally. - b. Genitalia dorsally. -c. Genitalia ventrally. - d. Spermathecae. - e. Spectacles-shaped sclerite. Scale bars 0.1 $\mathrm{mm}$.
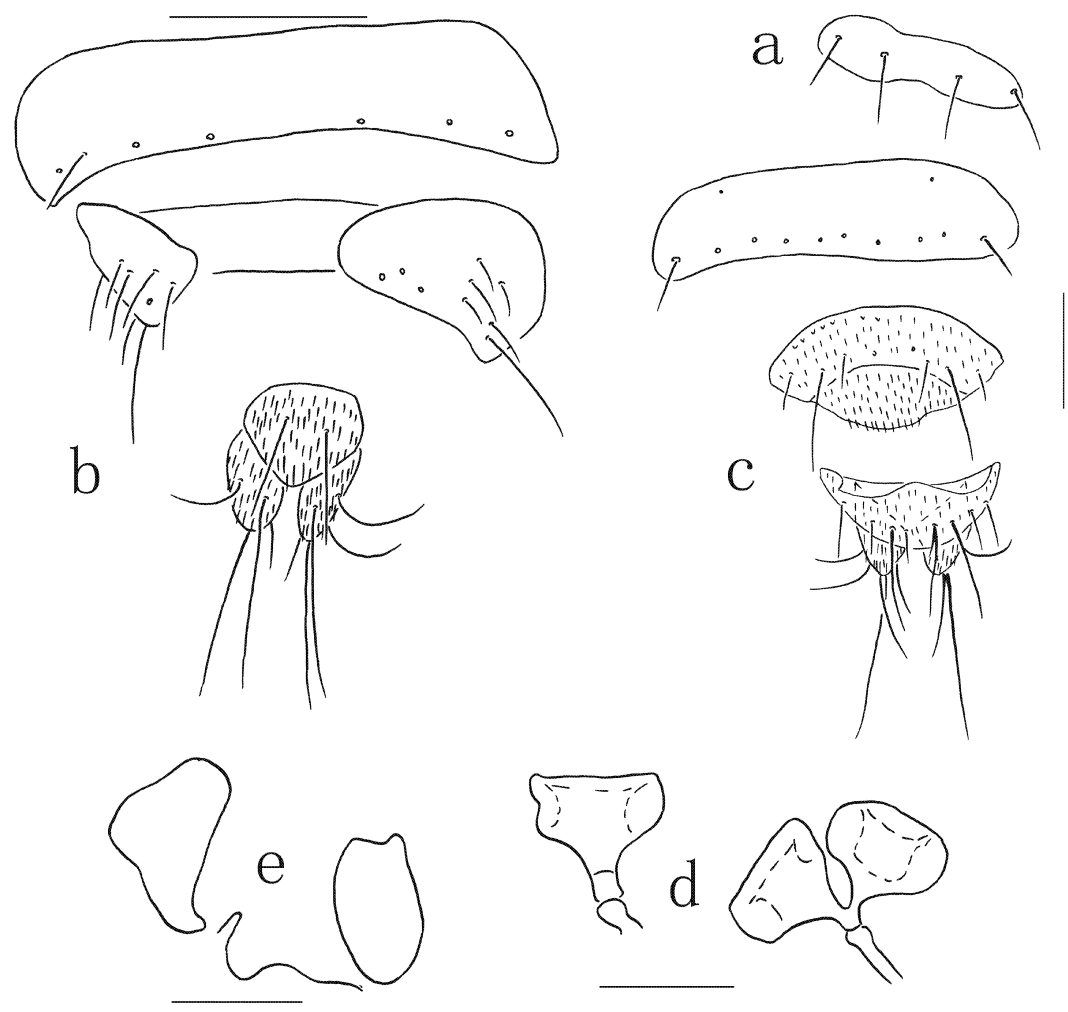

seta and 1 small setula between laterobasal and apical scutellar marginal setae. Mid tibia proximally with a short anterodorsal seta and a small anterodorsal seta in front of it, distally with a short anterodorsal and a long dorsal and a short posterodorsal setae, dorsal seta less than twice as long as posterodorsal seta, male without ventral seta except for short ventroapical seta (female with ventral seta below middle). Wing pale brown, veins brown. C extending slightly beyond $\mathrm{R} 4+5$. Second costal sector 0.9 times as long as third, crossveins $\mathrm{r}-\mathrm{m}$ and $\mathrm{dm}$-cu separated by 2.5 times the length of dm-cu. R 2+3 slightly straight on basal half, but very slightly bent up to $\mathrm{C}$ on apical half. R 4+5 slightly straight. Cell dm with appendages of $\mathrm{M}$ and $\mathrm{CuA}_{1}$, but indistinct. Alula small and narrow, apically blunt. Syntergite $1+2$ (Fig. 1f) darkly pigmented except for anteromedially pale and membranous, its length half at middle as long as darkly pigmented part; tergite 3 darkly and uniformly pigmented, slightly narrower and subequal in length to syntergite at middle. Synsternite 1+2 (Figs. 1g, 2a) narrow and wide, darkly and uniformly pigmented, with several medium long setae.
Male genitalia. Sternite 5 (Fig. 1e) posteromedial area densely setose, about 5 rows, posterior row of setae long (sometimes posterior row of long setae bent internally); sternite 6 simple. Surstylus (Fig. 1d) rectangular, laterally setulose at base; ventrally with small anterior and posterior processes, their apices slightly pointed (particularly anteroventral process sometimes spinelike). Basiphallus (Fig. 1a) square. Postgonite (Fig. 1a) slightly slender, preapically with 2 setulae. Distiphallus (Fig. 1a, 1h) dorsally with 4 pairs of processes in lateral view, not toothed.

Female genitalia. Tergite 8 (Fig. 2b) dorsomedially membranous, laterally widened and darker. Additional sclerite not visible. Tergite 10 (Fig. 2b) setose and with 2 long setae. Sternite 8 (Fig. 2c) setose and with 2 distinctly long setae, posterior half pale and densely setose. Sternite 10 (Fig. 2c) setose except for 2 anterior parts membraneous and bare, with 2 distinctly long setae. Spectacles-shaped sclerite (Fig. 2e) with oval rings. Spermathecae (Fig. 2d) shorter and wider.

Etymology. The specific name is from Latin for quadruplets and refers to 4 paired parts on 


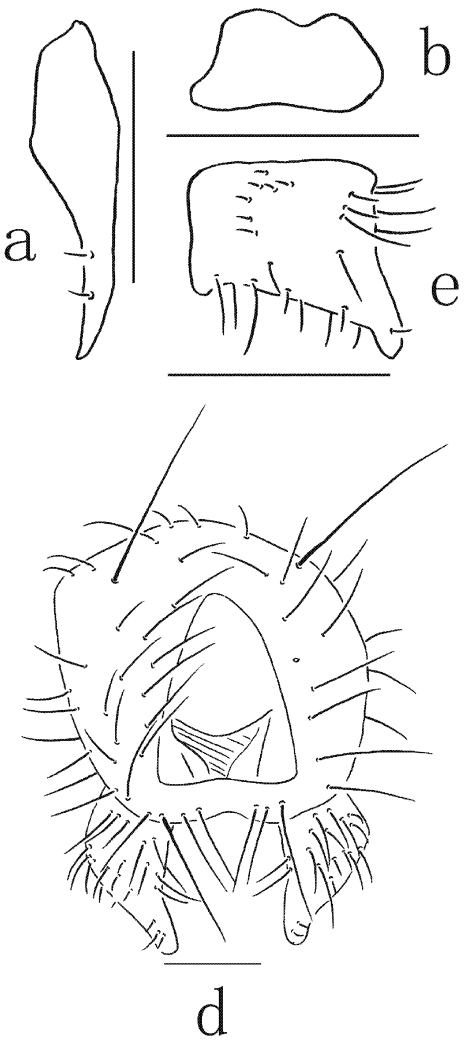

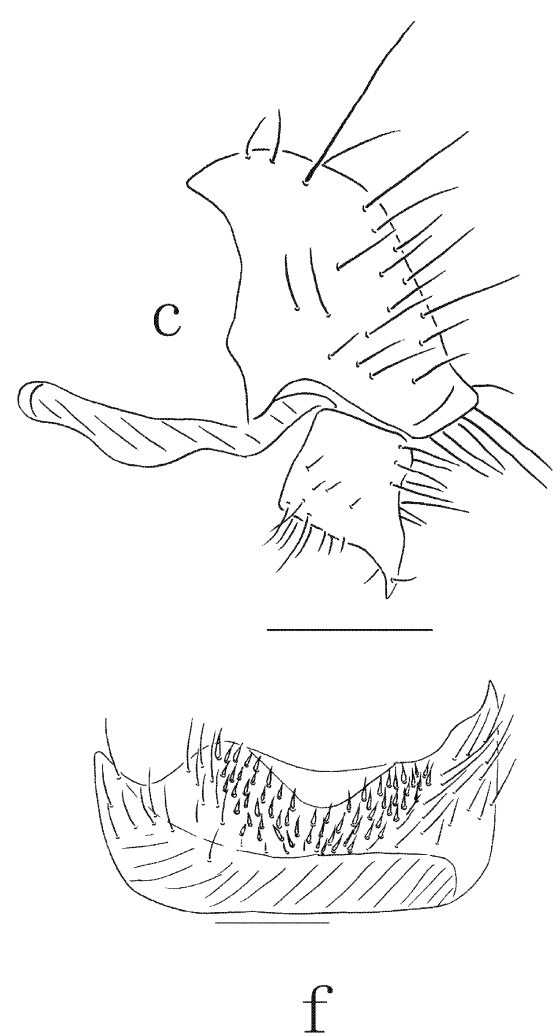

Fig. 3. Pullimosina (P.) heteroneura (Haliday), male, from Jiangxi, China. - a. Postgonite laterally. - b. Basiphallus laterally. $-\mathrm{c}$. Genitalia laterally (left side). - d. Genitalia caudally. - e. Surstylus laterally (right side). $-\mathrm{f}$. Sternite 5 ventrally. Scale bars $0.1 \mathrm{~mm}$. male distiphallus, forming 4 pairs of processes in lateral view (Fig. 1a, 1h).

Distribution. China: Liaoning Province.

Remarks. The new species resembles Pullimosina (Dahlimosina) yukonensis Marshall, 1986 in the external characters, male and female genitalia. It can be easily distinguished from the latter by body colour shining black; distiphallus with 4 paired parts; surstylus laterally with dense and long setulae, ventrally with an apically pointed, spine-like, anterior process; female tergites 1-5 without a large membranous area; female sternite 8 posterior half membranous and densely setose.

\subsection{Pullimosina (Dahlimosina) heteroneura (Haliday, 1836) (Fig. 3)}

Limosina heteroneura Haliday, 1836: 331.

Material examined. China: Jiangxi Province, Mt. Guan, Dong River, 114²3'3.4’'E, 28 25' 9.4’'N,
430 m, 31.III.2009 1 ๙ Lingli Yi \& Yi Li leg.; Jiangxi Province, Mt. Matou, $117^{\circ} 10^{\prime} 36.4^{\prime \prime} \mathrm{E}$, 2750'3.2”N, $410 \mathrm{~m}, 10 . \mathrm{IV} .20091 \AA$ Malaise trap.

Distribution. Afghanistan, Algeria, Andorra, Argentina, Australia, Austria, Belgium, Bermuda, Bulgaria, Cape Verde Is., Canada, China (Jiangxi), Cyprus, Czech Republic, Ecuador, Denmark, Egypt, Estonia, Finland, France, Greece, Gough I, Hungary, Iceland, Israel, Italy, Japan, Latvia, Lebanon, Lithuania, Macedonia, Malta, Mexico, Morocco, Netherlands, New Zealand, Norway, Poland, Portugal, Romania, Russia, Slovakia, Slovenia, South Africa, Spain, Sweden, Switzerland, Tajikistan, Taiwan, Tunisia, Uganda, United Arab Emirates, USA.

Remarks. The species is firstly recorded for Continental China. Some characters were found different from the description in Roháček's (1983) European revision as follows: Head: Interfrontal setae in 4 pairs, posterior second longer and cruciate, posterior first and third long and subequal in length, anterior first shortest, or all 
Fig. 4. Pullimosina (Pullimosina) meta Su, male, from Yunnan, China. - a. Mid tibia laterally.- b. Mid tibia dorsally. - c. Aedeagus and associated parts, laterally. - d. Genitalia laterally. $-\mathrm{e}$. Genitalia caudally. $-\mathrm{f}$. Surstylus laterally. - g. Sternite 5 ventrally. Scale bars
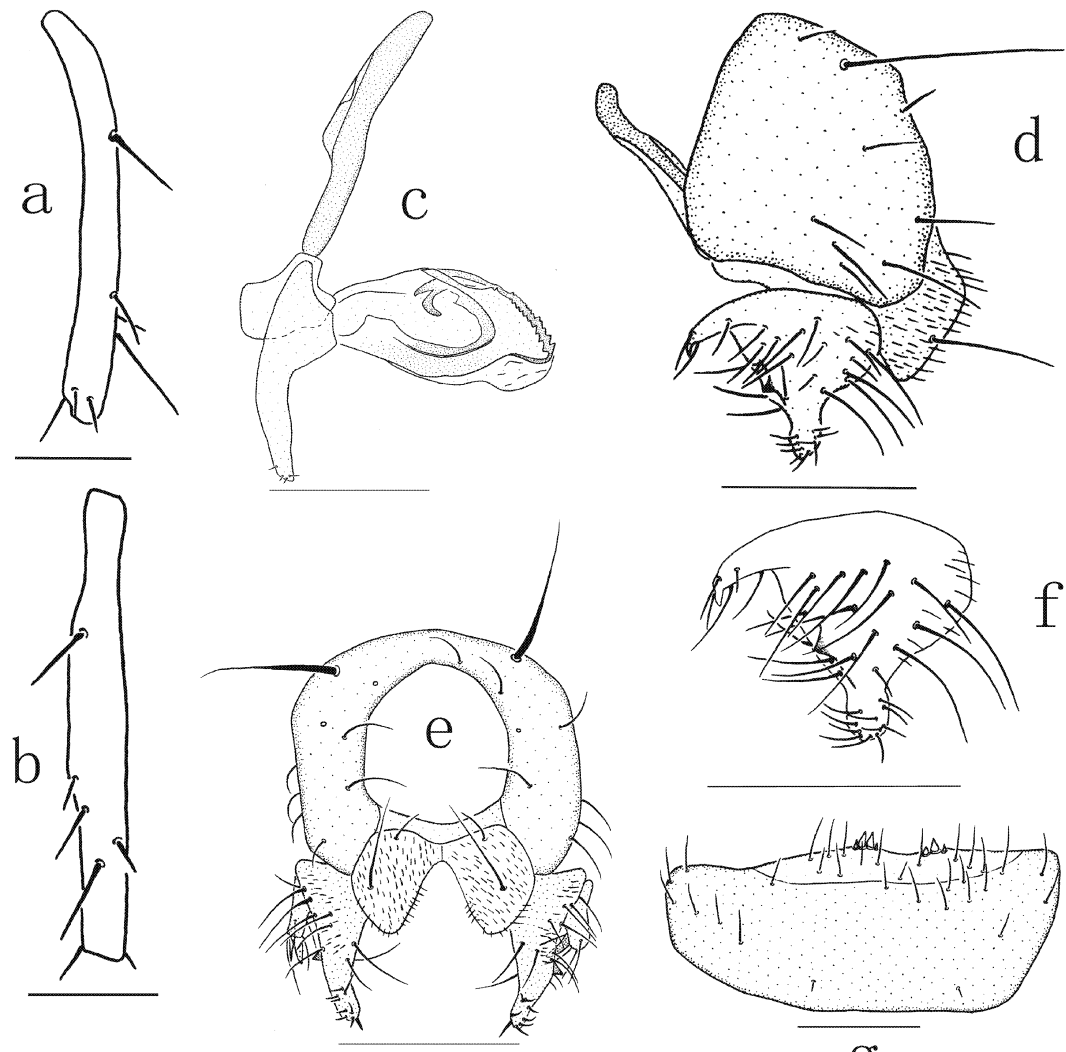

$0.1 \mathrm{~mm}$.

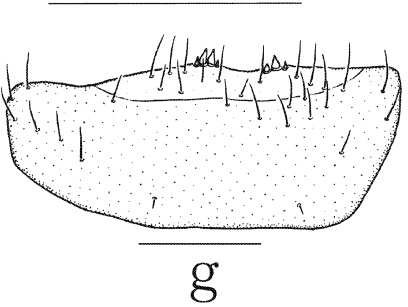

interfrontals subequal in length. Thorax: Acrostichal setulae in 4 rows in front of suture. Male genitalia: Sternite 6 with 2 small processes. Surstylus (Fig. 3c, 3e) usually symmetrical, but sometimes asymmetrical, surstylus in one side with an apically blunt, anteroventral process (usually without anteroventral process).

\subsection{Pullimosina (Pullimosina) meta Su, 2011} (Fig. 4)

Pullimosina (Pullimosina) meta Su, 2011: 9394, 197.

The original description is in Chinese, hence given here in English.

Material examined. China: Yunnan Province, Mt. Laifeng, 98²9'14.9'E, 250'55.9'N, 7.VIII.2009 1 ^ Jianfeng Wang \& Lixin Su leg.; Jiangxi Province, Mt. Guan, 114'33'6.3'E, 2829'59'N, 450-470 m, 9.V.2009 1 ð Zheping Yu \& Lingli Yi leg.

Description. Body length 1.6-1.5 mm, wing length 1.3-1.4 mm. General colour brownish black to black; head black; halter stem and knob brown; legs almost brownish black except for trochanters, basal parts of tibiae and tarsi, light brown. Head: Postvertical setae small. 3 interfrontal setae, middle one longest and strongly cruciate. Eye large, its height about 3 times as long as genal height. Aristal hairs 1.5-2 times as long as basal width of arista.

Thorax: Throacic chaetotaxy: 1 long postpronotal seta; 2 dorsocentral setae, anterior dorsocentral seta 0.5 times as long as prescutellar dorsocentral seta, prescutellar dorsocentral seta slightly shorter than scutellar length; acrostichal setulae in 6-8 rows in front of suture. Prescutellar acrostichal seta enlarged, but distinctly shorter and thinner than anterior dorsocentral seta. Scutellum with 4 marginal setae, without additional setulae, laterobasal marginal seta 0.6 times as long as apical marginal seta. Fore femur with a distinct row of posteroventral setae, enlarged and elongate in distal half. Mid tibia (Fig. 4a, 4b) 

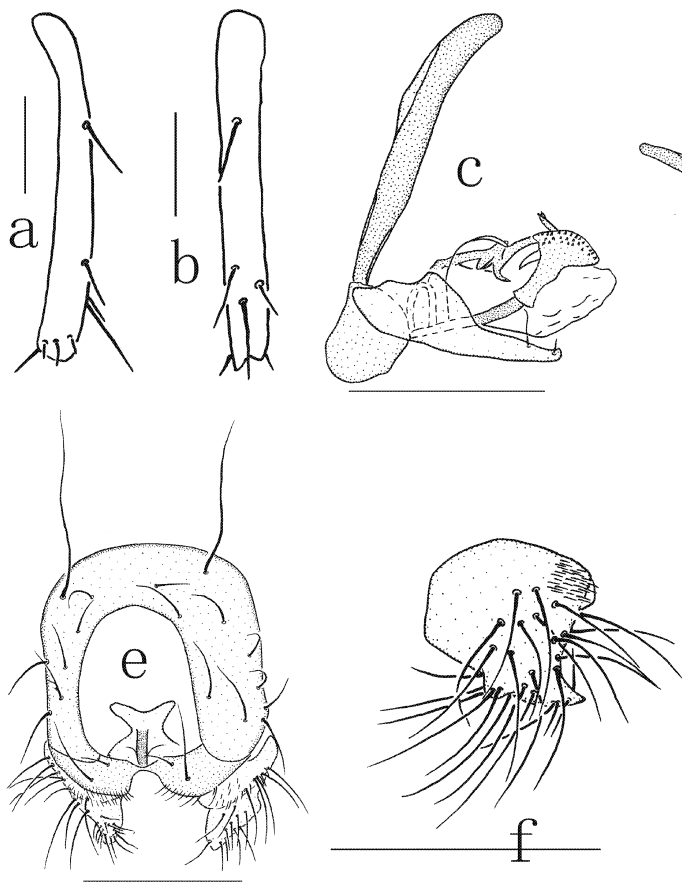

proximally with a slightly long anterodorsal seta, distally with a short anterodorsal and a longer dorsal and a short posterodorsal setae, dorsal seta distinctly more than twice as long as posterodorsal seta, male without ventral seta except for short ventroapical seta. Mid femur with a weak row of posteroventral setae. Wing pale brown, veins brown. $\mathrm{C}$ extending distinctly beyond $\mathrm{R}$ $4+5$. Second costal sector 0.8 times as long as the third, crossveins $\mathrm{r}-\mathrm{m}$ and $\mathrm{dm}$-cu separated by 3.3 times the length of dm-cu. R $2+3$ slightly straight on basal half, apically distinctly bent up to $\mathrm{C}$. $\mathrm{R}$ $4+5$ distinctly bent up to $C$. Cell dm with appendages of $\mathrm{M}$ and $\mathrm{CuA}$. Alula small and narrow, apically blunt. Syntergite $1+2$ anteromedially lightly pigmented, its length $2 / 5$ at middle as long as darkly pigmented part; tergite 3 darkly and uniformly pigmented, slightly narrower and shorter than syntergite. Synsternite 1+2 light yellow. Sternite 3 shining black except for anteriorly with semicircular area light yellow.

Male genitalia. Sternite 5 (Fig. 4g) posteromedial area membranous, its posteromedial portion with 2-3 spines on right side and 4 spines on left side. Sternite 6 with a small, cone-like process. Epandrium (Fig. 4d, 4e) with long dorso-

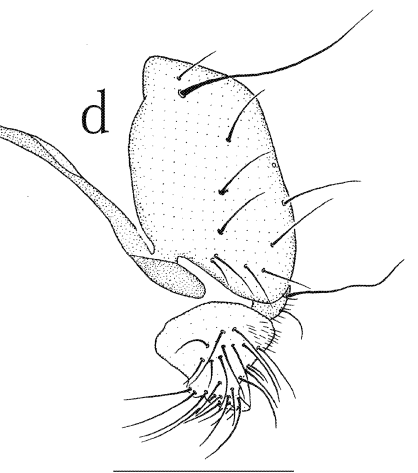

Fig. 5. Pullimosina (Pullimosina) vulgesta Roháček, male, from Ningxi, China. - a. Mid tibia laterally.- b. Mid tibia dorsally. $-\mathrm{C}$. Aedeagus and associated parts, laterally. - d. Genitalia laterally. - e. Genitalia caudally. - f. Surstylus laterally. - g. Sternite 5 ventrally. Scale bars $0.1 \mathrm{~mm}$.

lateral setae. Cercus (Fig. 4d, 4e) with a long seta. Surstylus (Fig. 4f) tripartite; lateral part with long setulae, anteromedial margin with a small, conelike process; anterodorsal margin of lateral part with a slender and long process (the character different from the original description), apically pointed and setulose; ventral margin of lateral part with a thick and short, setulose process. Basiphallus (Fig. 4c) rectangular. Postgonite (Fig. 4c) basal half broadened, apical half slender, its apex with small setulae. Distiphallus (Fig. 4c) distal sclerite toothed.

Female. Unknown.

Distribution. China: Jiangxi Province, Yunnan Province.

Remarks. The species resembles Pullimosina (Pullimosina) mcalpinei Marshall, 1986 in face and gena colour, interfrontals, sternite 6 , male surstylus. It can be easily diffenentiated from the latter by prescutellar acrostichal seta distinctly shorter and thinner than anterior dorsocentral seta; male sternite 5 posteromedial area with 2 clusters of spines, including 4 and 2-3 spines respectively; male surstylus anteromedial margin of lateral part with a cone-like process; distiphallus with toothed distal sclerite. 


\subsection{Pullimosina (Pullimosina) vulgesta Roháček, 2001 (Fig. 5)}

Pullimosina (Pullimosina) vulgesta Roháček, 2001: 474.

Pullimosina (Pullimosina) moesta: Roháček, 1983: 106-107; Roháček, 1985: 117, 120-121; Su, 2011: 95-96, 198.

Material examined. China: Jilin Province, Mt. Changbai, $128^{\circ} 3^{\prime} 39^{\prime \prime} \mathrm{E}, 42^{\circ} 0^{\prime} 4.8^{\prime}{ }^{\prime} \mathrm{N}, 900-1,100$ m, 30.VII.2008 4 $\widehat{\partial}$ Lixin Su leg.; Sichuan Province, Mt. Gongga, 10152'35.9'E, 29³5' 41.9"N, 1,500 m, 2.VIII.2004 2 ठे Gे Guangchun Liu leg.; Yunnan Province, Mt. Laifeng, 98 29'14.9'"E, 250'55.9'N , 1,750-1,950 m, 70.VIII.2009 $2 \widehat{\partial} \widehat{\partial}$ Lixin Su leg.; Ningxia Province, Mt. Liupan, Guamagou, $106^{\circ} 27^{\prime} 48^{\prime \prime} \mathrm{E}$, 35'51'26.1'”N, 2,000-2,250 m, 6.VII.2008 1 ठै Lixin Su leg.; Jiangxi Province, Mt. Guan, 114'23'3.4'”, $28^{\circ} 25^{\prime} 9.4^{\prime} ’ \mathrm{~N}, \quad 450-470 \mathrm{~m}$, 9.V.2010 4 ठठ Zheping Yu \& Lingli Yi leg.; Jiangxi Province, Jian County, Shuangiang, 114'30'19.3"E, 26⒌'43.1'N, $174 \mathrm{~m}, 17 . \mathrm{V}$. $20091 \delta$ Lingli Yi leg.; Jiangxi Province, Mt. Wuyi, $117^{\circ} 48^{\prime} 50.4^{\prime \prime} \mathrm{E}, 27^{\circ} 59^{\prime} 56.7^{\prime}$ 'N $1,170 \mathrm{~m}$, 18.VII.2009 2 ठै $\widehat{o}$ Malaise trap.

Distribution. Andorra, Austria, Belgium, China (Jiangxi, Jilin, Ningxia, Sichuan, Yunnan), Czech Republic, Denmark, Finland, France, Germany, Great Britain, Hungary, Ireland, Italy, Japan, Latvia, Montenegro, Nepal, Netherlands, ?North Korea, Norway, Poland, Portugal, Russia, Slovakia, Slovenia, Spain, Sweden, Switzerland.

Remarks. Some characters were found different from the description in Roháček's (1983) European revision as follows: Head: Interfrontal setae in 3-4 pairs, middle 1 pair or 2 pairs longest and strongly cruciate. Thorax: Acrostichal setulae in 6-9 rows in front of suture. Syntergite $1+2$ anteromedially pale and membranous, V-shaped (specimens from Sichuan Province deep, while specimens from other Provinces slightly shallow). Male genitalia: Sternite 5 (Fig. $5 \mathrm{~g}$ ) posteromedial area with $2-3$ spines. Sternite 6 with a small, cone-like process.

\subsection{Key to the genus Pullimosina Roháček from China}

1. Interfrontal setae subequal in length; scutellum also with some additional setulae in addition to the 4 marginal setae; wing with short $\mathrm{C}$ extended beyond R 4+5; male sternite 5 (Fig. 1e) posteromedial area setose

$P$. (D.) quadripulata $\mathrm{Su} \mathbf{s p .}$.

- Middle one of interfrontals longest and strongly cruciate; scutellum only with 4 marginal setae; wing with long $\mathrm{C}$ extended beyond R $4+5$; male sternite 5 posteromedial area with spines or spine-like setae 2

2. Wing distance between $\mathrm{r}-\mathrm{m}$ and $\mathrm{dm}-\mathrm{cu}$ shorter than dm-cu; male sternite 5 (Fig. 3f) posteromedial area with numerous spine-like setae $\quad P$. (P.) heteroneura (Haliday)

- Wing distance between $\mathrm{r}-\mathrm{m}$ and $\mathrm{dm}$-cu longer than dm-cu; male sternite 5 posteromedial margin with spines

3. Male sternite 5 (Fig. 4g) posteromedial margin with 7 small spines $\quad P$. (P.) meta $\mathrm{Su}$

- Male sternite 5 (Fig. 5g) posteromedial margin with 2 or 3 small spines

$$
\text { P. (P.) vulgesta Roháček }
$$

Acknowledgements. We thank Prof. Maoling Sheng, the Forest Pest Control Station of the State Forestry Administration, China, for his generous loan of specimens, and also Mr. Yifan Liu, University of Illinois at Urbana-Champaign, U. S. A., for providing valuable references. This work was supported by the National Nature Science Foundation of China (No. 30770252, No. 31071965).

\section{References}

Haliday, A. H. 1836: British species of the Dipterous tribe Sphaeroceridae. - Entomological Magazine 3: 315336.

Hayashi. T. 2006: The genus Pullimosina Roháček (Diptera, Sphaeroceridae) from Japan. - The Japan Society of Medical Entomology and Zoology 57(4): 265-272.

Marshall, S. A. 1986: A revision of the Nearctic species of the genus Pullimosina (Diptera, Sphaeroceridae). Canadian Journal of Zoology 64: 522-536.

Marshall, S. A., Roháček, J., Dong, H. \& Buck, M. 2011. The state of Sphaeroceridae (Diptera: Acalyptratae): a world catalog update covering the years 2000-2010, with new generic synonymy, new combinations, and new distributions. - Acta Entomologica Musei Nationalis Pragae 51(1): 217-298. 
Roháček, J. 1983: A monograph and re-classification of the previous genus Limosina Macquart (Diptera, Sphaeroceridae) of Europe. Part II. - Beiträge zur Entomologie, Berlin 33: 3-195.

Roháček, J. 1985: A monograph and re-classification of the previous genus Limosina Macquart (Diptera, Sphaeroceridae) of Europe. Part IV. - Beiträge zur Entomologie, Berlin 35: 101-179.

Roháček, J. 2001: The type material of Sphaeroceridae described by J. Villeneuve with lectotype designations and nomenclatural and taxonomic notes (Diptera). Bulletin de la Société entomologique de France 105(5) (2000): 467-478.
Roháček, J., Marshall, S. A., Norrbom, A. L., Buck, M., Quiros, D. I. \& Smith, I. 2001: World catalog of Sphaeroceridae (Diptera). — Slezské zemské Museum, Opava. 414 pp.

Smith, I. P. \& Marshall, S. A. 2004: A review of the New World genus Pterogramma Spuler and a review of the Pterogramma sublugubrinum group (Diptera: Sphaeroceridae: Limosininae). - Contributions in Science, Natural History Museum of Los Angeles County 499: $1-163$.

Su, L.-X. 2011: Lesser Dung Flies. — Liaoning University Press, Shenyang, China. 229 pp. 